Randomised controlled trial

\section{Collaborative care improves clinical outcomes for adolescents with depression treated in primary care}

\author{
10.1136/ebmed-2014-110108
}

\section{Janine Archer}

University of Manchester, School of Nursing, Midwifery \& Social Work, Manchester, UK

Correspondence to: Dr Janine Archer, University of Manchester, School of Nursing, Midwifery \&t Social Work, Jean McFarlane Building (Room 6.304), 0xford Road, Manchester M13 9PL, UK; Janine.archer@manchester.ac.uk

Commentary on: Richardson LP, Ludman E, McCauley E, et al. Collaborative care for adolescents with depression in primary care: a randomized clinical trial. JAMA 2014;312:809-16.

\section{Context}

Depression in adolescence is common worldwide and although it is treatable, only a minority receive evidence-based interventions. ${ }^{1}{ }^{2}$ A recent report by the WHO identifies depression as the leading cause of illness and disability in adolescents and highlights the importance of a coordinated approach to care. ${ }^{3}$ A Cochrane review has shown collaborative care interventions to improve depression in adults treated in primary care, but few studies have examined whether these interventions are effective in adolescents. ${ }^{4}$ This randomised controlled trial examines whether collaborative care interventions adapted for adolescents with depression improve outcomes compared with usual care.

\section{Methods}

Adolescents aged 13-17 years with depression were recruited from nine paediatric and family medicine units in the Group Health system located in three urban areas of Washington State in the USA between April 2010 and March 2011. Exclusions included non-English speaking, suicidal plan or recent attempt, bipolar, drug/alcohol misuse, seeing a psychiatrist, and developmental delay. Participants were block randomised using computer algorithms generated off-site, to either collaborative care or usual care using blocks of four within each of four strata defined by sex and age.

The Reaching Out to Adolescents in Distress intervention studied in the trial was an adapted version of the Improving Mood-Promoting Access to Collaborative Treatment model. ${ }^{5}$ Adaptations included developmentally sensitive material, involvement of parents, and choice of treatment and follow-up contacts. Collaborative care interventions, delivered by trained depression case managers during a 12-month period, included an initial education and engagement session and choice of antidepressant medications, brief cognitive-behaviour therapy, or both. Enhanced usual care consisted of informing the adolescents, parents and primary care clinician of depression test results, encouraging follow-up and recommending treatment. A stepped care algorithm was developed for those not meeting improvement criteria.

The primary outcome of interest was change in depressive symptoms from baseline to 12 months as measured by the Child Depression Rating Scale-Revised (CDRS-R; score range 14-94). Secondary outcomes included depression response (defined as a greater than $50 \%$ decrease in
CDRS-R score), depression remission, and functional status. Outcomes were assessed at 6 and 12 months by blinded research staff.

\section{Findings}

One hundred and one adolescents were enrolled and randomised to the intervention $(n=50)$ or control $(n=51)$ groups. There were no significant baseline differences between the two groups. At 12 months, participants in the intervention group had a 9.4 point greater decrease in mean CDRS-R scores of depressive symptoms compared with the control group (95\% CI -15 to $-3.8, p=0.001$ ). Participants in the intervention group were also more likely than participants in the control group to achieve depression response $(67.6 \%$ vs $38.6 \%, \quad \mathrm{OR}=3.3,95 \%$ CI 1.4 to 8.2 ; $\mathrm{p}=0.009$ ) and remission ( $50.4 \%$ vs $20.7 \%, 0 R=3.9,95 \%$ CI 1.5 to 10.6 ; $\mathrm{p}=0.007$ ) by 12 months. Both groups experienced improvement in functional status with no significant differences between groups.

\section{Commentary}

The study highlights the importance of addressing adolescent depression, which has been linked to increased mortality and morbidity. As such, this is a timely trial, given the global drive to improve the health and well-being of adolescents.

The authors' approach of adapting a previously established intervention to meet the particular needs of adolescents, including the involvement of parents, is welcome. Parents and caregivers have an important role to play in supporting adolescents with depression and should be engaged at all stages of assessment, diagnosis and treatment. ${ }^{6}$

The results of the trial suggest that collaborative care can improve depression in adolescents and that the intervention can feasibly be implemented in the primary care setting. Of importance is that the study demonstrates improved access and adherence to evidence-based interventions, key factors in addressing the increasing issue of adolescent depression. It is unfortunate that funding cuts resulted in a smaller sample size which, as the authors identify, may have resulted in decreased statistical power.

Further research is required to support the current findings, especially in non-Caucasian and non-English-speaking populations. As further studies are conducted, meta-regression to identify 'active ingredients' will refine the intervention and improve cost-effectiveness.

\section{Implications for practice}

Despite the acknowledged limitations, the findings of this study suggest that mental health services for adolescents with depression can be integrated into primary care with improved depression outcomes. Further research is required before widespread adoption of the collaborative care interventions for adolescents with depression, particularly in more diverse populations.

Competing interests None.

Provenance and peer review Commissioned; internally peer reviewed.

\section{References}

1. Thapar A, Collishaw S, Pine DS, et al. Depression in adolescence. Lancet 2012;379:1056-67.

2. Cummings JR, Druss BG. Racial/ethnic differences in mental health service use among adolescents with major depression. J Am Acad Child Adolesc Psychiatry 2011;50:160-70.

3. WHO. Health for the world's adolescents. A second chance in the second decade. Geneva: World Health Organization, 2014.

4. Archer J, Bower P, Gilbody S, et al. Collaborative care for depression and anxiety problems. Cochrane Database Syst Rev 2012;10:CD006525.

5. Unutzer J, Katon W, Callahan CM, et al. Collaborative care management of late-life depression in the primary care setting: a randomized controlled trial. JAMA 2002;288:2836-45.

6. NICE. Depression in children and young people identification and management in primary, community and secondary care. National Institute for Health and Care Excellence clinical guideline 28, 2005. 